\title{
Emphysème lobaire géant chez un nourrisson traité en urgence
}

\section{Giant Lobar Emphysema in an Infant treated Urgently}

\author{
O. Alaoui \\ (C) SFMU et Lavoisier SAS 2017
}

Un nourrisson âgé d'un mois de sexe masculin, sans antécédent pathologique notable, a été admis en urgence pour une détresse respiratoire d'installation brutale. L'examen a trouvé un nourrisson dyspnéique avec un hémithorax gauche distendu peu mobile, hypersonore, tympanique à la percussion, une diminution des murmures vésiculaires et des bruits du cœur déviés vers la droite. Une radiographie thoracique (Fig. 1A) avait montré une hyperclarté homogène intéressant l'hémithorax gauche avec élargissement des espaces intercostaux, abaissement de la coupole diaphragmatique homolatérale et une déviation du médiastin vers le côté controlatéral. Une TDM thoracique (Fig. 1B) a été réalisée, confortant le diagnostic d'emphysème lobaire géant gauche. Le nourrisson a été admis en urgence pour une thoracotomie de sauvetage vu l'aggravation clinique rapide. L'exploration chirurgicale a trouvé un emphysème lobaire géant supérieur gauche. Une lobectomie supérieure gauche a été réalisée. Les suites opératoires étaient simples avec une bonne évolu- tion clinique et radiologique pour un recul de deux ans (Fig. $1 \mathrm{C})$. L'emphysème lobaire géant est une malformation pulmonaire rare caractérisée par une distension progressive d'un lobe en raison d'un obstacle bronchique incomplet [1]. En période postnatale, la distension exagérée des voies aériennes par un effet de valve peut conduire à un syndrome de détresse respiratoire aiguë, imposant une prise en charge urgente, diagnostique et thérapeutique [2].

\section{Références}

1. Hadchouel-Duvergé A, Lezmi G, de Blic J, Delacourt C (2012) Congenital lung malformations: natural history and pathophysiological mechanisms. Rev Mal Respir 29:601-11

2. Hall NJ, Chiu PP, Langer JC (2016) Morbidity after elective resection of prenatally diagnosed asymptomatic congenital pulmonary airway malformations. Pediatr Pulmonol 51:525-30

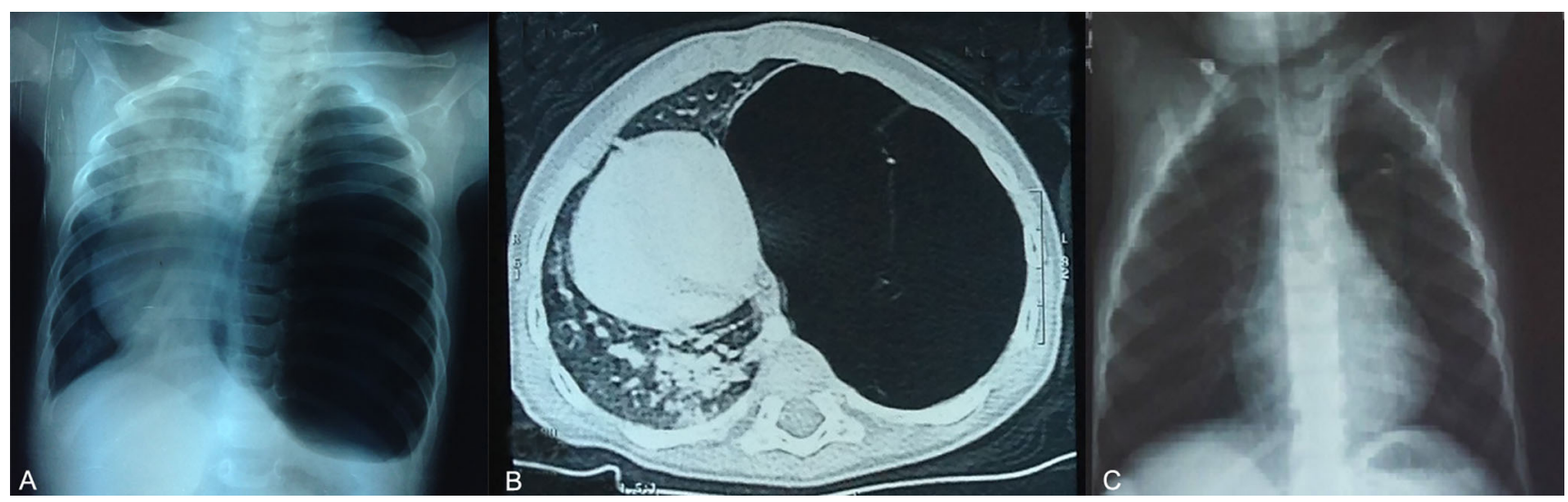

Fig. $1 \mathrm{~A} /$ radiographie thoracique de face montrant l'emphysème lobaire géant gauche ; B/ TDM thoracique montrant l'emphysème lobaire géant gauche $; \mathrm{C} /$ radiographie thoracique de face pour un contrôle avec deux ans de recul

O. Alaoui $(\bowtie)$

Service de chirurgie pédiatrique, CHU Hassan II, Fès, Maroc

e-mail : othmanechirped@gmail.com

Service de chirurgie pédiatrique, CHU Hassan II, route Sidi

Harazem, Fès, Maroc 